\title{
Lumbar scoliosis caused by dural ectasia
}

\begin{abstract}
Introduction: Dural ectasia is a rare menigeal alteration, which over $80 \%$ of cases related to Neurofibromatosis type I.

Case presentation: A 44-year-old female diagnosed with neurofibromatosis type I, who presented with severe lumbago and progressive worsening deformity in the coronal plane. She had no neurological deficit. In CT evaluation, we could diagnose destruction of multiple vertebral bodies and scoliosis, induced by giant dural ectasia.

Conclusion: Although the literature recommends conservative treatment for dural ectasia, in this case, due to refractory pain, scoliosis and the imminent risk of vertebral collapse, surgical treatment was chosen.
\end{abstract}

\author{
Volume 5 Issue 3 - 2018
}

\section{Cleiton Dias Naves, Luís Eduardo Carelli Teixeira da Silva, Caíque Jauhar de Castro, Marcelo Glauber da Silva Pereira, Renato João Muniz Teixeira}

Surgeons at the National Institute of Traumatology and Orthopedics of Brazil (INTO), Brazil

\begin{abstract}
Correspondence: Cleiton Dias Naves, Surgeons at the National Institute of Traumatology and Orthopedics of Brazil (INTO), Rio de Janeiro, Brazil, Tel 5521988477272 , Email dr.cleitonnaves@gmail.com, ORCID 0000-0002-25457942
\end{abstract}

Received: May 0I, 2018 | Published: May 30, 2018

\section{Review}

The literature estimates that between 70 and $80 \%$ of dural ectasia occur in patients with Neurofibromatosis type I, also known as von Recklinghausen's disease. ${ }^{1}$ Autosomal dominant disease of changerelated etiology on chromosome $17(17 \mathrm{q} 11.2)$, in the gene $\mathrm{NF}^{2}$ responsible for the production of the protein neurofibromin, ${ }_{3}$ being inherited in $50 \%$ of the cases, and by spontaneous mutation in the other 50\%. It presents an incidence of 1: 3000, varying the statistic according to the region of the planet. ${ }^{4}$ According to the NIH - National Institute of Health - the diagnosis is established by the presence of two or more characteristics within the seven: (1) six or more coffeewith-milk stains, (2) two or more neurofibromas; or a plexiform neurofibroma, (3) inguinal or axillary ephelides, (4) Lisch nodules in the iris, (5) optic glioma, (6) first-degree relative carrying the disease, and (7) typical skeletal alteration of the disease spectrum. ${ }^{5}$ With a lower incidence, dural ectasia may also be present in patients with Marfan syndrome, Ehlers-Danlos syndrome and ankylosing spondylitis. ${ }^{6}$

\section{Case}

A 44-year-old female patient affected by neurofibromatosis type I and arterial hypertension. Refers lumbar pain of severe intensity 7 years ago with progressively deterioration 8 months ago determining significant functional limitation, incapacitating the patient to perform their daily activities. Negative paraesthesia or radiated paresis for lower limbs. Symptom control uses increasing daily opioid doses.

\section{Physical exam}

She wanders freely, with lumbar pain.

Sensitivity preserved in all dermatomes;

Degree $\mathrm{V}$ force in all myotomes corresponding to the roots of the lumbosacral plexus;
Normal sphincter function bilaterally present patellar and aquileu Reflexes;

Frankel Scale: E (normal)

Electroneuromyography: Normal

Displays the following images: (Figure 1) (Figure 2).

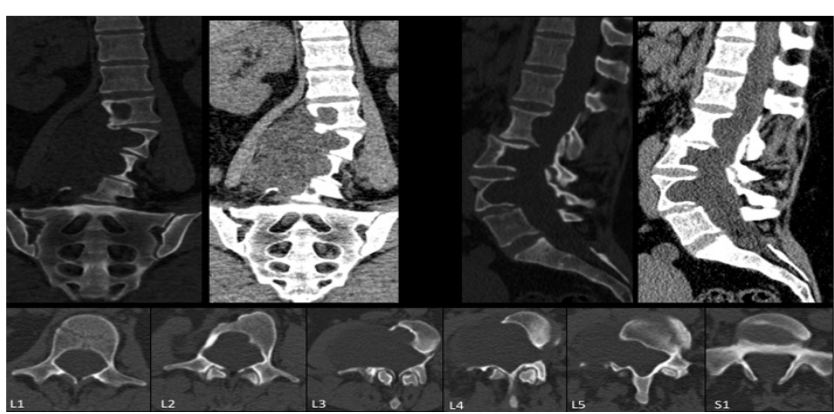

Figure 1 Computed tomography images.

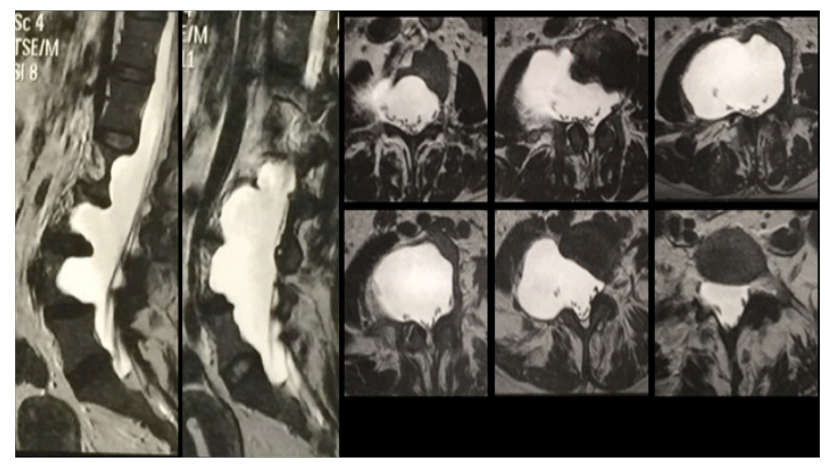

Figure 2 Magnetic resonance images. 
Through the diagnosis of dural ectasia with destruction of several vertebral bodies and imminent risk of vertebral collapse at minimal trauma, surgical treatment was proposed.

Planning has become a challenge, aiming at preserving neurological structures and achieving stabilization through arthrodesis. The case was discussed in the clinical session of the Institute and it was decided by a double approach (anterior+posterior) on different days. The first surgical time involved a retroperitoneal anterior approach with pararetal access

Vascular and Neurological structures were visualized, osteoarticular structures were identified with gross anatomical alterations, and discectomy and stabilization were performed with a Harms basket with autologous bone graft in the intersomatic spaces.

This surgical time was successfully completed without any complications. After a week, the second surgical time was performed by posterior approach, in which a dural sac with gross posterior deviation was identified and visualized at the level of the spinous processes (Figure 3). There was a adventitious dural sac lesion of the during the dissection, being found duramater at the level of the spinous processes; durorrafia was performed. The arthrodesis of Hibbs and Moe (inter- and supra-facetary), instrumentation with T11-S1iliac pedicle screws and postero-lateral arthrodesis were performed between the transverse processes, always using autologous bone graft. As routine conduct in cases of durorrafia, it was intended to remain 7 days with the bed at zero degree.

After this period, the patient could wander again and was discharged (Figure 4) (Figure 5).

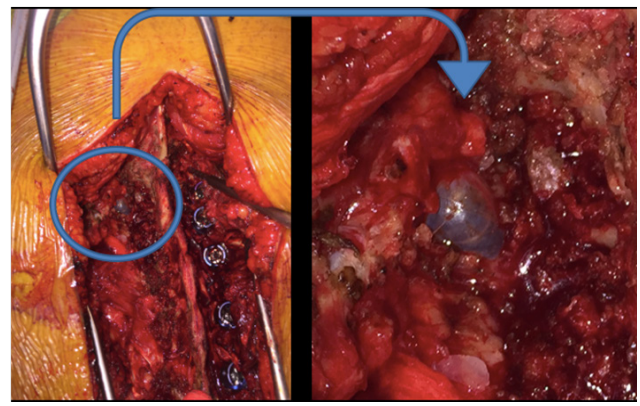

Figure 3 Intra-operative dural sac at the level of the spinous process Postoperative images

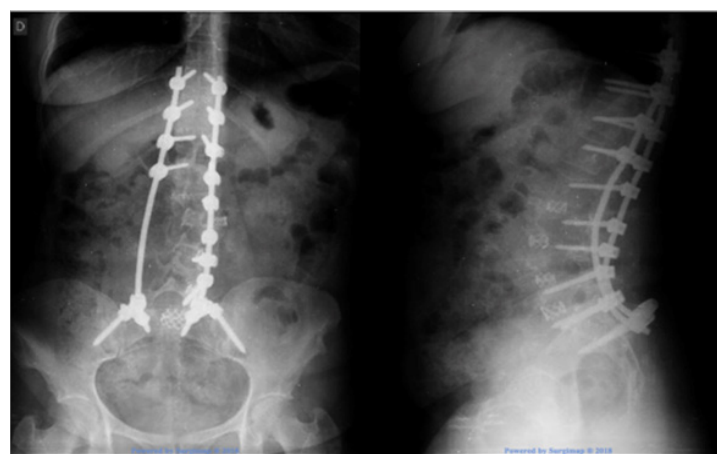

Figure 4 Postoperative radiographs.

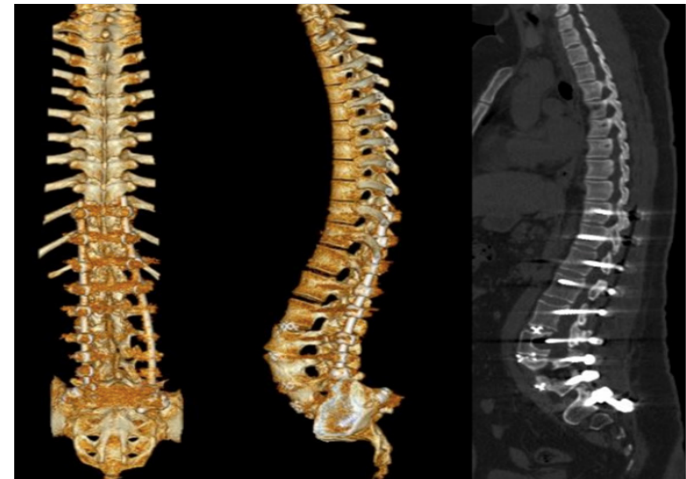

Figure 5 Postoperative computed tomography.

\section{Discussion}

Dural ectasia usually occurs in the thoracic or lumbar spine, causing chronic pain, and with the progression of the disease can cause radiculopathy, myelopathy and even equine tail syndrome, due to the adhesion of the medullary cone to the meningeal wall; and in some cases there may be low-pressure cerebrospinal fluid syndrome. ${ }^{7}$

A study presented cut-off values for the definition of dural ectasia in patients with adolescent idiopathic scoliosis (AIS), with a cutoff value of 0.84 and 0.58 for the Dural Sac Ratio for T5 and L5 respectively; measured by the ratio between the diameter of the dural sac and the diameter of the vertebral body. It's concluded that morphological changes related to dural ectasia are more frequent in patients with AIS than in controls subjects. ${ }^{8}$

The literature emphasizes the need for a dual access approach for the surgical treatment of dystrophic scoliosis in neurofibromatosis's patients. A series of cases suggests the use of hybrid instrumentation composed of a pedicle screw associated to sublaminar ligature in patients with a marked alteration in the sagittal plane (hyperkyphosis) associated with scoliosis. It also showed that patients with kyphoscoliosis presented greater intraoperative bleeding, greater blood loss, longer surgical time and greater loss of correction at follow up. ${ }^{9}$

A recent systematic review of the literature has indicated that in general the treatment of dural ectasia, alteration of the meningeal type III according to the classification of Jorg Klekamp, is a conservative treatment, ${ }^{10}$ mainly due to the impossibility of reconstruction of the dural sac and the complications involved with the alteration of the dynamics of the spinal fluid when restricting its continent, in a chronic course pathology. However, in this case, considering the degree of vertebral involvement, the secondary deformity caused by the disease and the imminence of a vertebral collapse, which would have catastrophic and irreversible consequences, the therapeutic possibilities were discussed and concluded that for this patient the surgical treatment would be the best option; for being able to perform the stabilization of the spine and treat scoliosis, performing correction in the sagittal and coronal alignment, optimizing the sagittal and coronal balance, restoring the biomechanics of the spine as close as possible to the physiological one.

\section{Conclusion}

At the last outpatient evaluation, with follow up of 2 years 
of surgery, the patient reported complete improvement of pain symptoms, return to her usual activities and a significant improvement in her quality of life. We understand that the surgical approach was determinant for symptomatic relief and prevention of presumable vertebral collapse.

\section{Acknowledgements}

None.

\section{Conflict of interest}

The author declares that there is no conflict of interest.

\section{References}

1. Razek AAKA. MR imaging of neoplastic and non-neoplastic lesions of the brain and spine in neurofibromatosis type I. Neurol Sci. 2018;39(5):821827.

2. Rosser TL, Vezina G, Packer RJ. Cerebrovascular abnormalities in a population of children with neurofibromatosis type 1. Neurology. 2005;64(3):553-555.

3. Gutmann DH, Parada LF, Silva AJ, et al. Neurofibromatosis Type 1: Modeling CNS Dysfunction. J Neurosci. 2012;32(41):14087-14093.
4. Gutmann DH. Ferner RE, Listernick RH, et al. Neurofibromatosis type 1 Nat Rev Dis Prim. 2017;3:17004.

5. Boyd KP, Korf BR, Theos A. Neurofibromatosis type 1. J Am Acad Dermatol. 2009;61(1):1-14.

6. Lee WJ, Park OJ, Won $\mathrm{CH}$, et al. Neurofibromatosis type 1 with dural ectasia. J Dermatol. 2012;39(7):655-656.

7. Liu CC, Lin YC, Lo CP, et al. Cauda equina syndrome and dural ectasia: Rare manifestations in chronic ankylosing spondylitis. $\mathrm{Br} \mathrm{J}$ Radiol. 2011;84(1002):123-125.

8. Abul-Kasim K, Overgaard A, Ohlin A. Dural ectasia in adolescent idiopathic scoliosis: Quantitative assessment on magnetic resonance imaging. Eur Spine J. 2010;19(5):754-759.

9. Koptan W, Elmiligui Y. Surgical correction of severe dystrophic neurofibromatosis scoliosis: An experience of 32 cases. Eur Spine J. 2010;19(9):1569-1575.

10. Klekamp J. A New Classification for Pathologies of Spinal Meninges, Part 1: Dural Cysts, Dissections, and Ectasias. Neurosurgery. 2017;81(1):2944. 